\title{
Low Xylitol Concentration induces Ultrastructural Cellular Changes in Streptococcus mutans and Streptococcus sobrinus
}

\author{
${ }^{1}$ Agnes de Fátima Faustino Pereira, ${ }^{2}$ Thiago Cruvinel da Silva \\ ${ }^{3}$ Maria Aparecida de Andrade Moreira Machado, ${ }^{4}$ Marília Afonso Rabelo Buzalaf
}

\begin{abstract}
Background: Xylitol is a sugar alcohol frequently used in the prevention of dental caries. However, the mechanism of action of xylitol on mutans streptococci is still unclear. This study evaluated the ultrastructural cellular changes in Streptococcus mutans and Streptococcus sobrinus after xylitol treatment.

Materials and methods: S. mutans ATCC 25175 and S. sobrinus ATCC 33478 were grown separately in $\mathrm{BHI}$ medium plus $0.2 \%$ sucrose at $37^{\circ} \mathrm{C}$ under microaerophilic conditions overnight. Then, planktonic cells were harvested by centrifugation, resuspended in a fresh medium and incubated with either $0.2 \%$ glucose or $0.2 \%$ xylitol solutions for 2 hours. To prepare bacterial cells for the evaluation of morphology of cellular walls and polysaccharide capsules, cultures were subsequently fixed with $2.5 \%$ glutaraldehyde, stained with ruthenium red, post-fixed with $1 \%$ osmium tetroxide, dehydrated in acetone, embedded in Epon LX112 and analyzed by a transmission electron microscopy.
\end{abstract}

Results: Bacterial cells incubated with glucose showed welldefined cellular walls and well-stained cytoplasmic membrane. Unlike, xylitol yielded diffuse cellular wall and irregular polysaccharide capsules in both microorganisms.

Conclusion: Low xylitol concentrations also can induce cellular damage to both $S$. mutans and S. sobrinus. Further studies are needed to provide more evidence on the anticariogenic effect of xylitol.

Keywords: Xylitol, Streptococcus mutans, Streptococcus sobrinus, Microscopy, Transmission.

How to cite this article: Pereira AFF, Silva TC, Machado MAAM, Buzalaf MAR. Low Xylitol Concentration induces

\footnotetext{
${ }^{1}$ Postdoctoral Fellow, ${ }^{2-4}$ Professor

${ }^{1}$ Hospital for Rehabilitation of Craniofacial Anomalies University of São Paulo, Bauru, São Paulo, Brazil

${ }^{2,3}$ Department of Pediatric Dentistry, Orthodontics and Public Health, Bauru School of Dentistry, University of São Paulo São Paulo, Brazil

${ }^{4}$ Department of Biological Sciences, Bauru School of Dentistry University of São Paulo, São Paulo, Brazil
}

Corresponding Author: Agnes de Fátima Faustino Pereira Postdoctoral Fellow, Hospital for Rehabilitation of Craniofacial Anomalies, University of São Paulo, Bauru, São Paulo, Brazil Phone: 0551432455229, e-mail: agnes_fatima@yahoo.com.br
Ultrastructural Cellular Changes in Streptococcus mutans and Streptococcus sobrinus. Int J Experiment Dent Sci 2015;4(1):1-3.

Source of support: São Paulo Research Foundation (FAPESP), São Paulo, Brazil (2008/08751-7).

\section{Conflict of interest: None}

\section{INTRODUCTION}

Streptococcus mutans and Streptococcus sobrinus have been strongly implicated as the main etiological agents in human dental caries. These microorganisms are able to form biofilm and produce weak organic acids, leading to mineral loss and progression of dental caries lesions. ${ }^{1}$

Mutans streptococci can incorporate but not metabolize xylitol, a five-carbon sugar alcohol frequently found in chewing gums and oral healthcare products. The consumption of xylitol-containing products might decrease the growth of mutans streptococci and its acid production; ${ }^{2}$ however, the mechanism of action of xylitol on pathogenic bacteria is still unclear. Theoretically, xylitol is phosphorylated, dephosphorylated and expelled from the cells without producing energy. ${ }^{3}$ This 'futile cycle' promotes the depletion of intracellular metabolic activity and a lower production of adherent extracellular polysaccharides and lipoteichoic acids, resulting in the formation of loosely attached biofilms to tooth surfaces. ${ }^{3}$

Tapiainen et $\mathrm{al}^{4}$ and Tuompo et $\mathrm{al}^{5}$ have reported respectively more diffuse and damaged Streptococcus pneumoniae cells and autolysis of $S$. mutans after treatment with xylitol solutions. The effectiveness of xylitol against microorganisms depends on its concentration. To our knowledge, however, the effects of $<0.38 \%$ xylitol on the ultrastructure of mutans streptococci have not yet been investigated. Besides, there is no evidence on the effect of xylitol on the cellular morphology of S. sobrinus. This study aimed to evaluate the effect of $0.2 \%$ xylitol on the ultrastructure of $S$. mutans and $S$. sobrinus.

\section{MATERIALS AND METHODS}

Laboratory of Microbiology of Bauru School of Dentistry, Bauru, Brazil. Pure stock solutions of S. mutans ATCC 25175 and S. sobrinus ATCC 33478 were grown 
separately in Brain Heart Infusion (BHI) Medium (Difco Laboratories, Detroit, MI, USA) plus 0.2\% sucrose at $37^{\circ} \mathrm{C}$ under microaerophilic conditions overnight $(\mathrm{OD} \approx 0.3-0.4$ at $650 \mathrm{~nm}$, Spectrophotometer Ultrospec 2000) (Pharmacia Biotech, Cambridge, England). Cultures were harvested by centrifugation and re-suspended in a fresh medium. Each cell suspension $(1 \mathrm{ml})$ was incubated with a final concentration of $0.2 \%$ glucose (control) or xylitol (test). Planktonic cells were incubated in 5\% $\mathrm{CO}_{2}$ at $37^{\circ} \mathrm{C}$ for additional 2 hours. Then, bacterial cells were harvested by centrifugation $(3,615 \mathrm{xg}, 10 \mathrm{~min})$ (Eppendorf, Hamburg, Germany) from a volume of $1.5 \mathrm{ml}$ and directly prepared to ultrastructural analysis by transmission electron microscopy.

To ultrastructural analysis, the bacterial pellets were fixed overnight at $78^{\circ} \mathrm{C}$ with $1 \mathrm{ml}$ of $2.5 \%$ glutaraldehyde in $0.1 \mathrm{M}$ phosphate buffer containing $100 \mathrm{ml}$ of ruthenium red (Sigma Chemical Co, St Louis, USA) for staining. It was then washed three times in phosphate buffer, postfixed in $1 \%$ osmium tetroxide, dehydrated in acetone and embedded in Epon LX112. Thin sections were cut with the ultramicrotome Leica Ultracut-UC2 (Leica Microsystems, Wetzlar, Germany) and examined with the transmission electron microscope Zeiss EM900 $(\mathrm{A}=30,000)$ (Zeiss, Munich, Germany) for a blinded examiner. One hundred cells of each microorganism were evaluated to describe the cellular morphology changes. All experiments were performed in triplicate.

\section{RESULTS}

Qualitative ultrastructural analysis of S. mutans and S. sobrinus after $0.2 \%$ xylitol and $0.2 \%$ glucose treatments are shown in Figures 1 and 2. Glucose solution did not yield morphological changes in both streptococci. Right side of Figures 1 and 2 show well-defined cell walls and well-stained cytoplasmic membrane of S. mutans and S. sobrinus respectively.

General morphology of bacterial cells was damaged for xylitol solutions. Streptococcus mutans and S. sobrinus treated with $0.2 \%$ xylitol showed more dispersed and unequal polysaccharide capsules, sloughing, intracellular vacuoles, and autolysis (left side of Figs 1 and 2). Xylitol made the cell walls of both microorganisms more diffuse and less defined when compared to control groups.

\section{DISCUSSION}

This study demonstrated that $0.2 \%$ xylitol yields more dispersed and unequal polysaccharide capsules, sloughing, intracellular vacuoles and autolysis in planktonic cells of S. mutans and S. sobrinus. Tuompo et $\mathrm{a}^{5}$ also reported cellular damages in $S$. mutans after incubation in $50 \mathrm{mg} / \mathrm{ml}$ xylitol solution. Tapiainen et $\mathrm{al}^{4}$ described similar effects of $0.5 \%$ xylitol on the ultrastructure of $S$. pneumoniae.

To compare the cellular morphology of bacteria from control and test groups, strains were respectively incubated in $0.2 \%$ glucose or $0.2 \%$ xylitol for 2 hours. This methodological approach were in accordance with Tapiainen et $\mathrm{al}^{4}$ microorganisms from control group were exposed to glucose based on previous results of Tuompo et $\mathrm{al}^{5}$ that demonstrated the absence of alterations in the ultrastructure of $S$. mutans after glucose and sucrose treatments. In addition, the authors described autolysis and intracellular vacuoles as common findings among bacterial cells treated with xylitol, which also can be observed in this study.

The intracellular accumulation of xylitol phosphate is responsible for inhibiting many intermediaries of

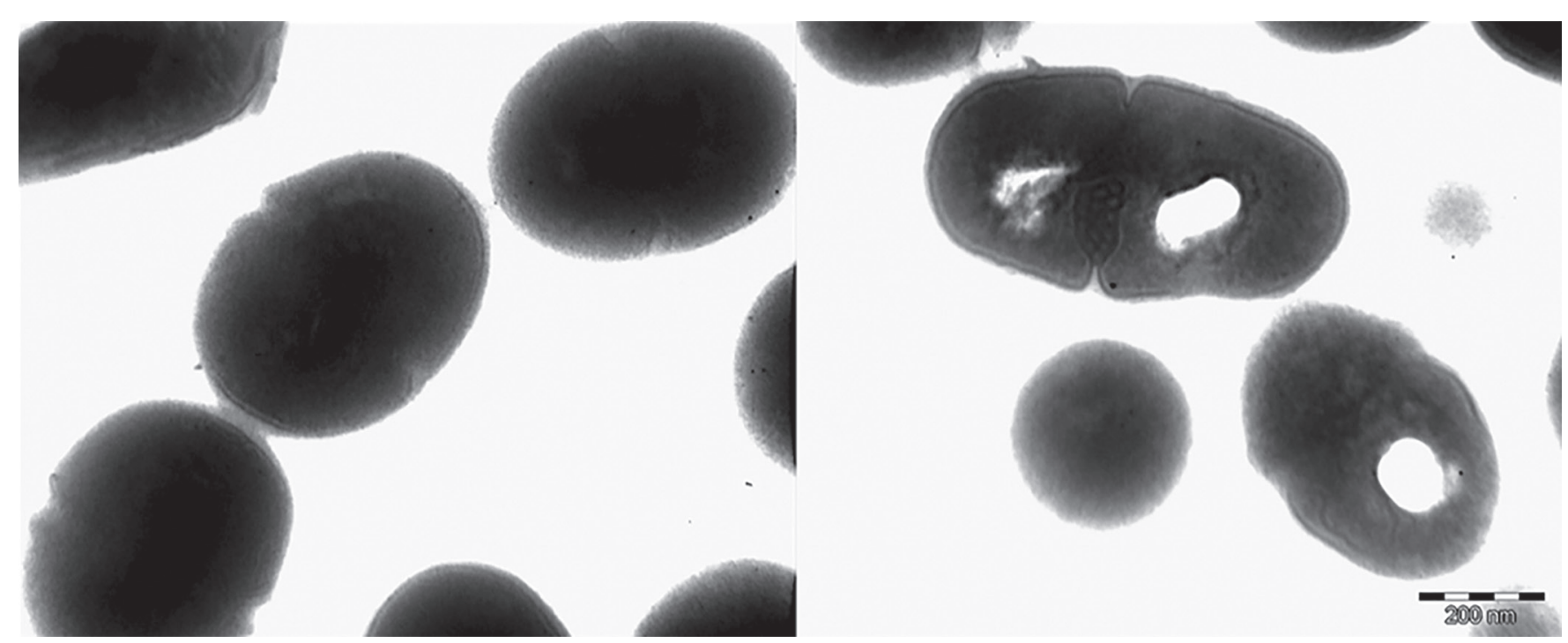

Fig. 1: Ultrastructural cellular morphology of S. sobrinus ATCC 33478 after $0.2 \%$ glucose (left side) and $0.2 \%$ xylitol (right side) treatments 

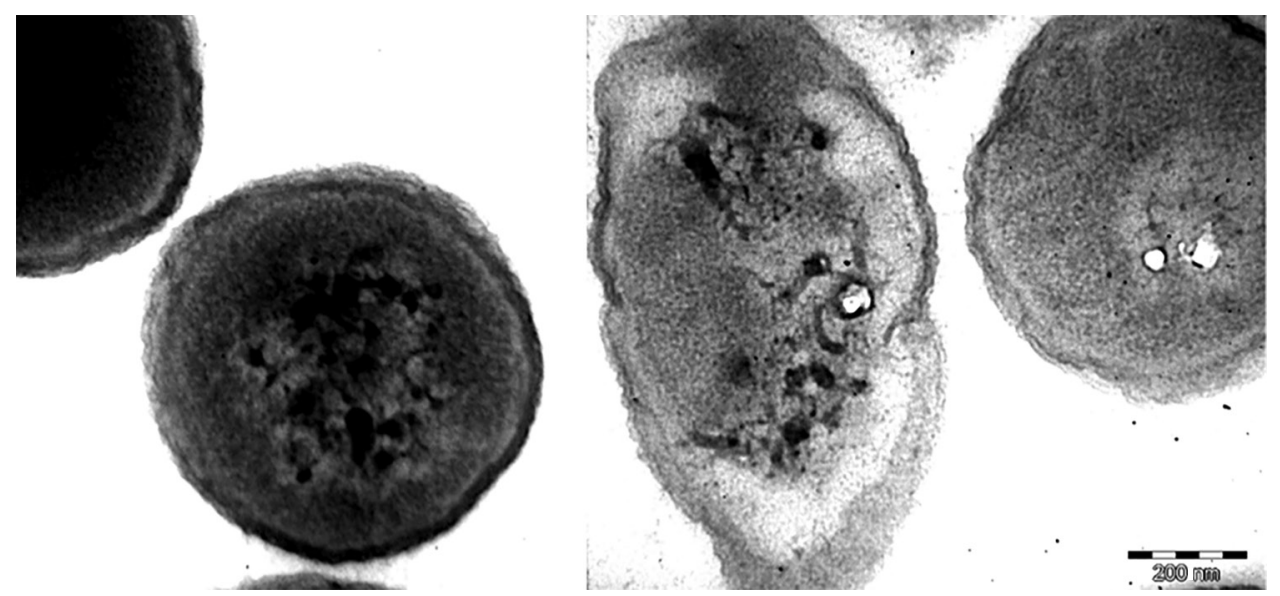

Fig. 2: Ultrastructural cellular morphology of S. mutans ATCC 25175 after $0.2 \%$ glucose (left side) and $0.2 \%$ xylitol (right side) treatments

glycolytic pathway, such as glucose-6-phosphate, fructose6-phosphate and glyceraldehyde-3-phosphate. ${ }^{3}$ These intermediaries are essential to enzymatic activation and, therefore, their inhibition prevents the metabolism of xylitol and energy production. Morphological cellular changes lead to reduced survival, virulence and adherence of microorganisms, ${ }^{6}$ which might explain the clinical benefits of xylitol on the prevention of dental caries. Söderling et $\mathrm{al}^{7}$ demonstrated a dose-dependent growth inhibition of $S$. mutans after exposure to xylitol.

Currently, the clinical efficacy of xylitol in the prevention of dental caries is related to the maintenance of minimum salivary levels $>1 \%$. However, this concentration can be detected in saliva for approximately 15 minutes after consumption of xylitol-containing products. ${ }^{8}$ The evidence that lower xylitol concentrations can affect ultrastructure of cariogenic microorganisms might promote the development of slow-release vehicles, which are able to maintain therapeutic xylitol concentrations in microenvironments, such as polymeric matrix and fluid of dental biofilm, for longer.

In summary, xylitol demonstrated its potential as a caries-preventive treatment by promoting ultrastructural cellular changes in planktonic cells of S. mutans and S. sobrinus. Further studies are needed to elucidate the effects of xylitol on the ultrastructure of bacterial cells from biofilms and possible mechanisms of antimicrobial action of xylitol on oral microorganisms.

\section{CONCLUSION}

However, dental caries is a multifactorial disease that starts with microbiological shifts within the complex biofilm (dental plaque) and this may be one of the important variables for dental caries risk assessment. ${ }^{9}$ Dentistry is varying with induction of modern science to practice dentistry. ${ }^{10}$

\section{ACKNOWLEDGMENTS}

This study was supported by São Paulo Research Foundation (FAPESP), São Paulo, Brazil. The authors thank Prof Dr Elliot Watanabe Kitajima, for his technical support in the transmission electron microscopy.

\section{REFERENCES}

1. Selwitz RH, Ismail AI, Pitts NB. Dental caries. The Lancet 2007;369(6):51-59.

2. Söderling EM. Xylitol, mutans streptococci, and dental plaque. Adv Dent Res 2009;21(1):74-78.

3. Assev S, Rölla G. Further studies on the growth inhibition of Streptococcus mutans OMZ 176 by xylitol. Acta Pathol Microbiol Immunol Scand 1986;94(2):97-102.

4. Tapiainen T, Sormunen R, Kaijalainen T, Kontiokari T, Ikäheimo I, Uhari M. Ultrastructure of Streptococcus pneumoniae after exposure to xylitol. J Antimicrob Chemother 2004;54(1):225-228.

5. Tuompo H, Mcurman JH, Lounatmaa K, Linkola J. Effects of xylitol and other carbon sources on the cell wall of Streptococcus mutans. Scand J Dent Res 1983;91(1):17-25.

6. Lee YE, Choi YH, Jeong SH, Kim HS, Lee SH, Song KB. Morphological changes in Streptococcus mutans after chewing gum containing xylitol for 12 months. Curr Microbiol 2009;58(4):332-337.

7. Söderling EM, Ekman TC, Taipale TJ. Growth inhibition of Streptococcus mutans with low xylitol concentrations. Curr Microbiol 2008;56(4):382-385.

8. Milgrom P, Lyl KA, Roberts MC, Rothen M, Mueller G, Yamaguchi DK. Mutans streptococci dose response to xylitol chewing gum. J Dent Res 2006;85(2):177-181.

9. Saini R. Optical detection of dental caries. Chron Young Sci 2011;2(1):61.

10. Saini R. Ozone therapy in dentistry: a strategic review. J Nat Sc Biol Med 2011;2(2):151-153. 